\title{
Relación entre perímetro braquial y algunos indicadores de crecimiento intrauterino*
}

\author{
Dr. lgnacio Sánchez D. ${ }^{1}$; Dr. Felipe Cavagnaro S.M.1: \\ Dr. José Luis Tapia I. ${ }^{2}$; Dra. Gabricla Juez G, ${ }^{2}$ \\ Mid-arm circumference and intrauterine growth
}

\begin{abstract}
Two hundred and seven healthy newborns, 35 to 42 wreeks of gestationl age (GA) determined by strict criteria, were prospectively studied. Mid arm circumference (MAC) in left arm was measured between 25 and 72 hours of age. Birth weight (BW), lenght ( $(\mathrm{L})$, and head circumference (HC) were obtained from each baby's codified chart. Ponderal index (PI) was calculated. Linear correlation (r) values were for MAC vs BW + 0.85; MAC vs L + 0.70; MAC vs GA $+0,48$; MAC vs PI +0.50 and $\mathrm{BW}$ vs $\mathrm{GA}+0.56$. In the MAC vs GA values there was also a great variability; specially over 38 weeks of GA. The same occurs for BW vs GA and Lvs GA. It is concluded that in this study, MAC correlated very well with $B W$ being useful to evaluate nutritionsl status. MAC showed a poor correlation with GA, which differs from previously published studies.
\end{abstract}

(Key words: Mid-arm circumference, head circumference, ponderal index, fetal growth, intrauterine growth retardation, fetal macrosomia.)

El perímetro braquial (PB) y la razón perímetro braquial/circunferencia de cráneo $(\mathrm{PB} / \mathrm{CC})$ han sido ampliamente utilizados en el lactante mayor de 3 meses y en el preescolar y escolar. Se ha demostrado que estas mediciones son buenos indicadores del estado de nutrición y que complementan a las más clásicamente empleadas, como el peso, talla, circunferencia craneana y la relación entre estas últimas ${ }^{1}$. Así tambiến, Frisancho ha publicado tablas patrón de referencia de crecimiento a partir del año de edad, utilizando pliegues cutáneos (tricipital) y perímetro braquial ${ }^{2}$.

El empleo de estas mediciones en el recjén nacido (RN), tendría la ventaja de identificar mejor a los nifios con alteraciones en el crecimiento intrauterino, que presentarán mayor riesgo en el período neonatal, y en segundo lugar de ser un método fácil y no invasivo de seguimiento del estado nutritivo postnatal en el $\mathrm{RN}$

1. Médico becado. Departamento de Pediatría. Pontificia Universidad Católica de Chile.

2. Médico. Unidad Neonatologia. Departamento de Pediatría. Pontificia Universidad Católica de Chile.

- Trabajo ptesentado en: XII Jormadas Chilenas de Pediatría, Viña del Mar, Chile, Noviembre 1987. JII Seminario Internacional de Cuidados Intensivos Neo. natales. Buenos Aires, Argentina, abril de 1988. prematuro, cuando las mediciones tradicionales pueden no ser un buen reflejo de este crecimiento.

Recientemente se han publicado curvas estándar de PB y la razón $\mathrm{PB} / \mathrm{CC}$ vs edad gestacional (EG) en el periodo neonatal ${ }^{3}$. Por ser éste un estudio extranjero, con otro tipo de población que la que atiende nuestra Unidad, y basados en la experiencia de las curvas de crecimiento intrauterino (CIU), publicadas en años recien$\mathrm{tes}^{4,5}$, consideramos de suma importancia validar una curva patrón propia, realizada en población sana, con la que se puedan comparar futuras mediciones; de aquí que el presente trabajo tiene como propósito, en primer lugar, comprobar si hay coincidencia con los estudios extranjeros, y de ser, asj servir como una herramienta útil en la evaluación nutricional de nuestros RN.

\section{MATERIAL Y METODO}

En la Unidad de Neonatología del Hospital Clínico de la Pontificia Universidad Católica de Chile se estudió en forma prospectiva a 207 RN sanos de 35 a 42 sernanas de edad gestacional. Se excluyó a los RN con antecedentes de enfermedades gestacionales o maternas (síndrome hipertensivo del embarazo, diabetes, colestasia intrahepática del embarazo, cardiopatías, infección urinaria); afecciones fetales (infecciones, malformacionos mayores, retardo de crecimiento, gemelares); trastornos placentarios (desprendimiento de placenta, 
placenta previa); edad gestacional dudosa (fecha de última regla no confiable, antecedentes de parto, aborto, lactancia 0 uso de anticonceptivos durante 6 meses previos al embarazo); madres fumadoras, consumidoras de drogas, con talla menor a $1,50 \mathrm{~m}$ y aumento de peso menor de $? \mathrm{~kg}$ en el embarazo.

Se midió el PB, entre las 25 y 72 horas de vida, en el punto medio del brazo izquierdo, manteniendo el codo en ángulo recto, considerando ur promedio de 3 mediciones; esto lo hicieron sólo dos investigadores, previo control de coincidencia de las medidas.

Se urilizó cinta de papel inextensible "Inser tape" (Laboratorio Ross) confeccionada con este propósito.

Las medidas antropométricas (peso, talla, circunferencia de cráneo) y la evaluación de la edad gestacional pediátrica se tomaron de la ficha codificada, que se utiliza en forma estándar en nuestra unidad, y que es la base de las curvas de crecimiento intrauterino 4,5 , Se calculó el Indice Ponderal (IP=peso x $100 /$ talla), por ser un buen indicador de la nutrición fetal. Los datos se analizaron en computador Apple Macintosh, obteniéndose promedios con desviación estándar y correlaciones entre las mediciones, con el programa "Statworks".

\section{RESULTADOS}

Se estudiaron $207 \mathrm{RN}$ que cumplieron los criterios de selección descritos. El peso promedio fue de $3.355 \pm 343 \mathrm{~g}(\tilde{\mathrm{X}} \pm \mathrm{DE})$, con rango 1.800 a $4.525 \mathrm{~g}$ y mediana $3.350 \mathrm{~g}$. Su talla fue $49,8 \pm$ $2,02 \mathrm{~cm}(\overline{\mathrm{X}} \pm \mathrm{DE})$, rango 41 a $55 \mathrm{~cm}$ y mediana $50 \mathrm{~cm}$. Las CC fueron $34,3 \pm 1,07 \mathrm{~cm}(\overline{\mathrm{X}} \pm \mathrm{DE})$, rango 30,5 a $37,3 \mathrm{~cm}$ y mediana de $34,3 \mathrm{~cm}$ y su indice ponderal $2,70 \pm 0,20(\mathrm{X} \pm \mathrm{DE})$, rango 2,0 $-3,37$ y mediana de 2,69. El PB fue de 9,86 \pm $0,66 \mathrm{~cm}(\bar{X} \pm D E)$, rango 7,6 a $12 \mathrm{~cm}$ y mediana $9,8 \mathrm{~cm}$.

En la tabla 1 se muestra la distribución de edad gestacional, sexo, promedio ( $\pm \mathrm{DE}$ ) de peso de nacimiento (PN) y el promedio $( \pm \mathrm{DE}$ ) de perímetro braquial de la población estudiada. Se observa un aumento progresivo en el PN a medida que avanza la EG hasta las 42 semanas en que disminuye levemente en relación a las 41 semanas de EG. El PB igualmente aumenta en concordancia con el PN y también muestra una leve disminución en la semana 42 de EG. La talla, $\mathrm{CC}$ e IP muestran esta misma tendencia.

En la figura 1 se muestra la correlación entre PB y PN en los $207 \mathrm{RN}$ entre 35 y 42 semanas; se observa claramente como el PB aumenta a medida que lo hace el PN; (r) $+0,85$ y coeficiente de determinación (det) 0,73 .

En la figura 2 se muestra la correlación entre el PB y EG. Se observa que ésta es directa y significativa, $r+0,48$ y det 0,23 , con una mayor
Tabla 1

Distribución de edad gestacional (EG), sexo, peso de nacimiento (PN) y perjinletro braquial (PB) de 207 recién nacidos

\begin{tabular}{|c|c|c|c|c|c|}
\hline \multirow[t]{2}{*}{ EG } & \multirow[t]{2}{*}{ No } & \multicolumn{2}{|c|}{ Sexo } & \multirow{2}{*}{$\begin{array}{l}P N(g) \\
\bar{X} \pm D E\end{array}$} & \multirow{2}{*}{$\begin{array}{l}\mathrm{PB}(\mathrm{cm}) \\
\bar{X} \pm \mathrm{DE}\end{array}$} \\
\hline & & $\mathbf{M}$ & $\mathrm{F}$ & & \\
\hline 35 & 8 & 5 & 3 & $2.550 \pm 327$ & $8,36 \pm 0,37$ \\
\hline 36 & 10 & 4 & 6 & $2.756 \pm 260$ & $9,04 \pm 0,56$ \\
\hline 37 & 10 & 6 & 4 & $3.035 \pm 182$ & $9,38 \pm 0,29$ \\
\hline 38 & 35 & 20 & 15 & $3.172 \pm 296$ & $9,72 \pm 0,52$ \\
\hline 39 & 51 & 24 & 27 & $3.466 \pm 388$ & $9,97 \pm 0,63$ \\
\hline 40 & 53 & 24 & 29 & $3.503 \pm 347$ & $10,04 \pm 0,57$ \\
\hline 41 & 30 & 12 & 18 & $3.612 \pm 402$ & $10,20 \pm 0,61$ \\
\hline \multirow[t]{2}{*}{42} & 10 & 6 & 4 & $3.432 \pm 312$ & $9,86 \pm 0,40$ \\
\hline & 20 & & & & \\
\hline
\end{tabular}

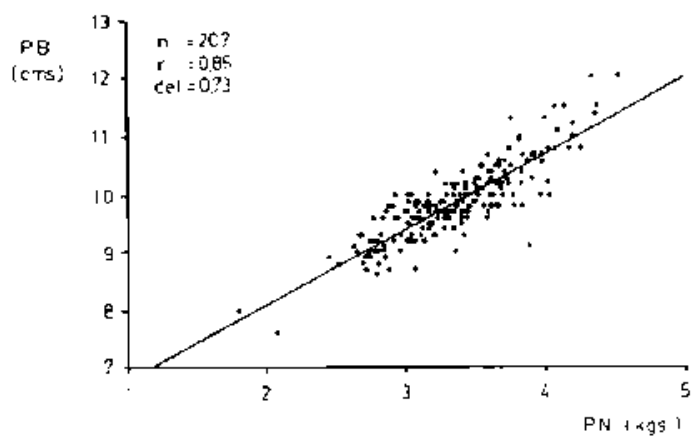

Figura 1. Cortelación entre Perímetro Braquial (PB) y Peso de Nacimiento (PN); 207 RN de 35 a 42 semanas EG.

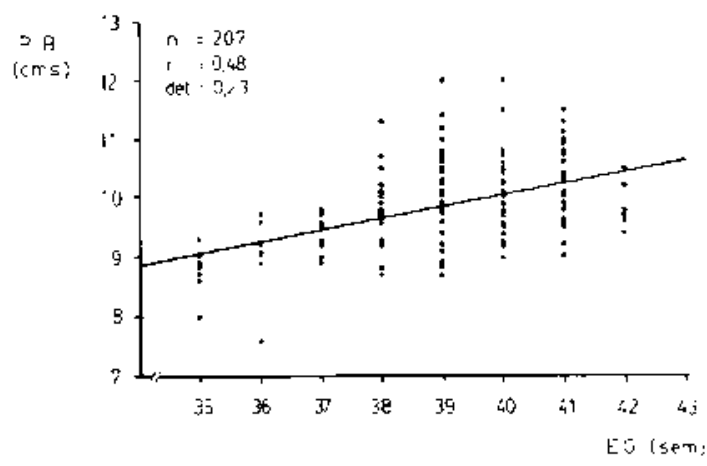

Figura 2. Correlación entre Perímetro Braquial (PB) y Edad Gestacional (EG); 207 RN de 35 a 42 semanas EG. 


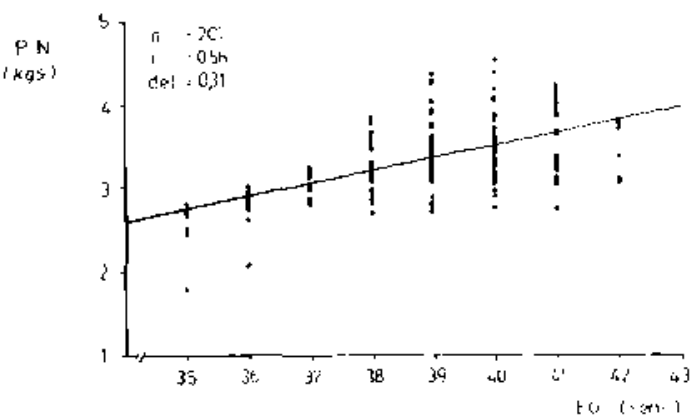

Figura 3. Correlación entre Peso de Nacimiento (PN) y Ldad Gestacional (EG); 207 RN de 35 a 42 semanas de EG.

variabilidad entre los valores, especialmente a partir de las 38 semanas.

En la figura 3 se muestra la correlación entre PN y EG. Esta es directa y significativa, $r+0,56$ y det 0,31 , similar a la anterior, manteniéndose la variabilidad mayor en los gnipos de edad gestacjonal más avanzada.

Otras correlaciones obtenidas, interesantes de destacar son:

$\mathrm{PB} / \mathrm{IP}, \mathrm{I}+0,42$ det $0,18 \mathrm{~PB} / \mathrm{T}, \mathrm{r}+0,70$; det 0,$49 ; \mathrm{PB} / \mathrm{CC}, \mathrm{r}+0,67 ;$ det 0,$45 ; \mathrm{T} / \mathrm{EG},+0,57$; det 0,$32 ; \mathrm{PB} / \mathrm{CC} / \mathrm{PN}, \mathrm{r}+0,68$ y $\mathrm{PB} / \mathrm{CC} / \mathrm{EG}, \mathrm{r}+$ 0,50 .

\section{DISCUSION}

Nuestro objetivo fue relacionar $\mathrm{PB} \mathrm{PB} / \mathrm{CC}$ con otros indicadores de crecimiento intrauterino, encontrándose muy buena correlación entre PB y peso de nacimiento, con minima variabilidad de los valores, a lo largo de las distintas edades gestacionales. Sin embargo, cuando se compara con la edad gestacional, la correlación es inferior, observándose gran dispersión de jos vajores, en especial hacia EG mayores. Estos resultados djfieren de estudios extranjeros ${ }^{3,6}$. Las causas de estas diferencias podrian estar en la población estudiada, constituida fundamentalmente por RN de término sanos, seleccionados según los criterios descritos.

El incremento del PB y de la razón $\mathrm{PB} / \mathrm{CC}$ con la edad gestacional, refleja principalmente el depósito de tejido graso en el brazo; se ha demostrado que el porcentaje de componente graso aumenta desde menos de $1 \%$ a las 24 semanas de
EG hasta 14\% en RN de término?. En varias publicaciones se utilizan distintas mediciones para separar el crecimiento de los componentes graso y muscular; ellas son muy útiles en la evaluación del creciniento intrauterino, ayudan a hacer mejores diagnósticos nutricionales y permiten alternativas de intervención en la prevención y eventual tratamiento $8,9,10$.

Alutores nacionales han demostrado que el PB y otras mediciones (pliegues cutáneos y cálculos de área grasa y magra) se correlacionan más con el estado de nutrición y la adecuación con la edad gestacional, que con el crecimiento en el tiempo, ya que serjan indices de nutrición fe$\mathrm{tal}^{11,12}$.

Las mediciones de $\mathrm{PB}$ y $\mathrm{PB} / \mathrm{CC}$ pueden ser de utilidad en la clasificación y seguimiento del es. tado de nutrición neonatal, especialmente en $\mathrm{RN}$ prematuros, ya que hay estudios que demuestran que el PB sería más especifico que otras mediciones, en discriminar $R N$ adecuados, pequeños $y$ grandes pata su edad gestacional que estén en riesgo de presentar distintos tipos de morbilidad en sus primeros días de vida ${ }^{6}$.

Sin embargo, en la población de RN mayores de 34 semanas, que aquí se presenta, el PB y $\mathrm{PB} / \mathrm{CC}$ no demostraron ser diferentes del $P N$ en su correlación con la EG. Además, PB y PB/CC tuvieron muy buena correlación con el PN, lo cual valoriza esta última medición en la evaJuación del estado nutritivo del RN sano en las EG estudiadas.

Es posible que el $\mathrm{PB}$ y la razón $\mathrm{PB} / \mathrm{CC}$ tengan mayor valor pronóstico que el PN en RN patológicos, como lo han comunicado otros autores ${ }^{6}$, lo que abre una interesante área de investigación futura.

\section{RESUMEN}

Se estudió en forma prospectiva a $207 \mathrm{RN}$ sanos, de 35 a 42 semanas, con estrictos criterios de selección; se midió el PB entre las 25 y 72 horas de vida, en el punto medio del brazo izquierdo. Se obtuvo la medidas de peso de nacimiento (PN), talla (T) y circunferencia cranea. na (CC) de la ficha codificada de la Unidad. Se calculó el índice ponderal (IP), y los valores promedios con desviación estándar de las mediciones, junto a la correlación lineal.

El PB X $=9,86 \pm 0,66 \mathrm{~cm}, P N \mathrm{X}=3.355 \pm$ $434 \mathrm{~g}$, Indice Ponderal (IP) $\mathrm{X}=2,70 \pm 0,20$. La 
correlación (r) $\mathrm{PB} / \mathrm{PN}+0,85 ; \mathrm{PB} / \mathrm{T}+0,70$; $\mathrm{PB} / \mathrm{EG}+0,48 ; \mathrm{PB} / \mathrm{IP}+0,50$ y $\mathrm{PN} / \mathrm{EG}+0,56$. En cuanto a $P B$ vs EG observamos que existe una gran variabilidad de los valores, en especial desde las 38 semanas en adelante, lo cual también ocurre con PN vs EG y T vs EG.

Se concluye que en este estudio el PB se correlaciona muy bien con el PN, siendo útil en la evaluación del estado nutritivo neonatal, y que al relacionarlo con edad gestacional, los valores presentan gran dispersión.

Estos resultados difieren de estudios extran. jeros.

\section{REFERENCIAS}

1. Kanawati A.A., Heddad N. McI,aren D.S. : The arm circumforence as a public health index of protein caloric malnutrition of early child-hood: preliminary results with mid-arm and muscle mid-arm circumferences used as nutritional screenning procedures for pre-school children in Lebanon. $\mathrm{J}$ Trop Pediatt 1969;15: 233-238.

2. Frisancho A.R.: New norns of upper limb fat and muscle areas for assessment of nutritional status. Am J Clin Nutr 1981, 34:2540-2\$45.

3. Sasanow S.R., Georgieff M.K and Pereira G.R.: Mid-arm circumference and mid-arm/head circumference ratios Standard curves for anthropometric assessment of neonatal nutritional status. $\mathrm{J}$ Pediatr $1986 ; 109: 311-315$.
4. Juez G., Ventura Juncá $P$, Lucero $E$.: Crecimiento intrauterino en un grupo seleccionado de Recién Nacidos Chilenos, ¿Subdiagnóstico de retardo de crecimiento en Chile? Rev Méd Chile 1984, 112: 759-764.

5. Ventura-Juncá P., Juez G., Lucero E.: Desnutrición intrauterina: Identificación de una nueva población de alto riesgo con una curva de peso chilena. Rev Méd Chile 1986; 114: 790-797.

6. Georgieff M.K., Sasanow S.R., Mammel M.C. and Pereira G.R: Mid-am circumference/head circumference ratios for identification of symptomatic LGA, AGA and SGA newborn infants. J Pediatr 1986; 109: 316-321.

7. Ziegler E.E., O'Donnal A.M., Netson S.E.: Body composition of the reference fetus. Growth 1976; 40: $239-245$

8. Frisencho A.R. Kleyman J.E. and Matos J.: Newborn body composition and its relations to linear growth. Am J Clin Nutr 1987; 30: 704-711.

9. Dauncey M.J., Gandy G. and Gairdner D.: Assessment of total body fat in infance from skinfold thickness measurements.

Arch Dis Child 1987;52: 233-227.

10. Enzi $G$, Zanardo $V$. , Cavette F. Inelmen EM. and Rubaltelli $F_{*}$ Intratuterine growth and adipose tissue development. Am J Clin Nutr 1981;34: $1785-1790$.

11. Krause S., Wenzel M.S. Krob K.: Influencia del estado nutricional materno sobre el tefido graso de? recién nacido. Rev Chil Pediatr 1978; 58: 203-206.

12. Kratuse S., Wenzel M.S., Grob K.: Composición corporal en Recién Nacidos de término. XII Jornadas Chileras de Pediatria, Vija del Mar, noviembre de 1987, NE-07, 155. 